\title{
Efficient Analysis of Multi-Resonant Periodic Structures for the Improved Analysis and Design of Reflectarray Antennas
}

Rafael Florencio

and Rafael R. Boix

\author{
José A. Encinar
}

\begin{abstract}
In order to design reflectarray antennas within reasonable CPU times, fast and accurate numerical tools for the analysis of periodic multilayered structures are required. In this paper the method of moments in the spectral domain (MoMSD) based on multilayered Green's functions (MGF) is applied to the analysis of periodic structures containing multilayered stacked patches in the unit cell. These multiresonant cells are potential elements for the design of reflectarray antennas. In the paper we show that the use of basis functions with edge singularities in the approximation of the current density on the patches leads to important computer memory and CPU time savings in the analysis of the periodic structures. Also, a rational fitting technique is introduced which makes it possible to obtain closed-form expressions for the scattering matrix of the periodic structures in terms of the dimension of the patches used to adjust the phase of the reflectarray elements.
\end{abstract}

\section{INTRODUCTION}

Printed reflectarrays are planar reflector antennas made of one or more layers of microstrip patch arrays. The microstrip patches of the arrays are conveniently tuned to produce a progressive phase shift of the reflected field. This makes it possible to collimate or to conform the radiated beam when the reflectarray is illuminated by a feed (usually a horn antenna), just as it happens with conventional reflectors [1]. A crucial stage in the design of reflectarray antennas made of patches of variable size is the determination of the sizes of the patches that lead to the appropriate reflection phases for the generation of a certain radiation pattern. When choosing the sizes of the patches, it is customary to assume that each patch is surrounded by an infinite periodic array of patches of the same size. This is known as the local periodicity assumption, and it makes it possible to design reflectarray antennas within reasonable CPU times [2]. In the design and optimization of reflectarray antennas under the local periodicity assumption, the electromagnetic analysis of the scattering of a plane wave by a periodic multilayered structure may be performed hundreds of thousands of times [3]. Since general purpose commercial software tools are not usually capable to carry out a fast analysis of such periodic structures, it is necessary to implement efficient home-made numerical tools for the analysis of the periodic structures.

In this paper, we will use the method of moments in the spectral domain (MoM-SD) [4] based on multilayered Green's functions (MGF) [5] for the analysis of the multilayered periodic structures that are required in the design of reflectarray antennas under the local periodicity assumption. In particular, we will deal with periodic structures containing multilayered stacked patches in the unit cell. We will show that the use of basis functions with edge singularities in the approximation of the current density on the patches [6] leads to important computer memory and CPU times savings in the analysis of the periodic structures. Also, a fitting technique will be introduced to obtain closed-form rational approximations for the scattering matrix of periodic arrays of patches in terms of the dimension of the patches that is used to adjust the phase of the reflectarray elements [7].

\section{EFFICIENT ANALYSIS OF MULTILAYERED PERIODIC STRUCTURES FOR REFLECTARRAY APPLICATIONS}

Figs. 1(a) and (b) show a multilayered periodic structure. The unit cell of the structure contains two stacked rectangular patches. The array of stacked patches is embedded in a multilayered substrate made of $N C$ layers of thickness $h_{i}(i=$ $1, \ldots, N C)$ and complex permittivity $\varepsilon_{i}=\varepsilon_{0} \varepsilon_{r i}\left(1-j \tan \delta_{i}\right)$. The multilayered substrate is limited below by a ground plane. The periodic structure is illuminated by a plane wave. Let $\mathbf{E}^{\mathrm{inc}}(x, y, z)$ be the electric field of the plane wave impinging on the periodic structure depicted in Fig. 1, and let $\mathbf{E}^{\text {ref }}(x, y, z)$ be the electric field of the reflected wave propagating in the specular direction. Let us define a scattering matrix $\overline{\mathbf{S}}_{X Y}$ that relates the complex amplitudes of the tangential components of $\mathbf{E}^{\mathrm{ref}}(x, y, z=0)$ and $\mathbf{E}^{\mathrm{inc}}(x, y, z=0)$ (i.e., the complex amplitudes of the tangential reflected and incident electric fields at the upper limit of the multilayered substrate) as shown 
below

$$
\left(\begin{array}{c}
E_{0 x}^{\mathrm{ref}} \\
E_{0 y}^{\mathrm{ref}}
\end{array}\right)=\overline{\mathbf{S}}_{X Y} \cdot\left(\begin{array}{c}
E_{0 x}^{\mathrm{inc}} \\
E_{0 y}^{\mathrm{inc}}
\end{array}\right)=\left(\begin{array}{cc}
S_{x x} & S_{x y} \\
S_{y x} & S_{y y}
\end{array}\right) \cdot\left(\begin{array}{c}
E_{0 x}^{\mathrm{inc}} \\
E_{0 y}^{\text {inc }}
\end{array}\right)
$$

The phase of the diagonal coefficients $S_{x x}$ and $S_{y y}$ are the key parameters when the unit cell of the periodic structure is employed in the design of a reflectarray antenna. The scattering matrix $\overline{\mathbf{S}}_{X Y}$ can be written as $\overline{\mathbf{S}}_{X Y}=\overline{\mathbf{S}}_{X Y}^{\mathrm{wd}}+\overline{\mathbf{S}}_{X Y}^{\mathrm{sc}}$, where $\overline{\mathbf{S}}_{X Y}^{\text {wd }}$ accounts for the effect of the multilayered substrate in the absence of the patches, and a scattering matrix $\overline{\mathbf{S}}_{X Y}^{\mathrm{sc}}$ which accounts for the effect of the patches. The matrix $\overline{\mathbf{S}}_{X Y}^{\mathrm{wd}}$ can be easily computed in terms of $4 \times 4$ transition matrices as shown elsewhere [8]. The computation of the matrix $\overline{\mathbf{S}}_{X Y}^{\mathrm{sc}}$ demands a larger CPU time, and can be carried out by means of the method of moments in the spectral domain (MoM-SD) [4] based on multilayered Green's function (MGF) [5]. In fact, the determination of the matrix $\overline{\mathbf{S}}_{X Y}^{\mathrm{sc}}$ requires to obtain the current density induced on the patches by the impinging wave. This current density is the solution of the following electric field integral equation (EFIE)

$$
\begin{array}{r}
\hat{\mathbf{z}} \times\left[\mathbf{E}^{\mathrm{wd}}\left(x, y, z=-s_{k}\right)\right. \\
+\sum_{l=1}^{2} \int_{-\infty}^{\infty} \int_{-\infty}^{\infty} \overline{\mathbf{G}}\left(x-x^{\prime}, y-y^{\prime}, z=-s_{k} \mid z^{\prime}=-s_{l}\right) \\
\left.\cdot \mathbf{J}^{l}\left(x^{\prime}, y^{\prime}\right) d x^{\prime} d y^{\prime}\right]=0
\end{array}
$$

$$
(0 \leq x \leq a ; 0 \leq y \leq b ; k=1,2)
$$

where $\mathbf{E}^{\mathrm{wd}}\left(x, y, z=-s_{k}\right)(k=1,2)$ is the electric field existing at the interface $z=-s_{k}$ of the multilayered substrate in the absence of the patches, $\mathbf{J}^{l}(x, y)(l=1,2)$ is a Floquetperiodic function that represents the induced current density on the patches located in the plane $z=-s_{l}$ of Fig. 1(a), and $\overline{\mathbf{G}}$ is the non periodic dyadic Green's function of the multilayered structure. In order to solve the EFIE (2), we will approximate $\mathbf{J}^{l}(x, y)$ as a summation of Floquet-periodic entire-domain basis functions as shown below

$$
\mathbf{J}^{l}(x, y)=\sum_{i=1}^{N_{b}} d_{i}^{l} \mathbf{J}_{i}^{l}(x, y) \quad(l=1,2)
$$

where the coefficients $d_{i}^{l}\left(i=1, \ldots, N_{b} ; \quad l=1,2\right)$ are unknown coefficients. When (3) is substituted into (2) and the Galerkin's version of the MoM is applied, a system of linear equations for the unknown coefficients $d_{i}^{l}$ is obtained. By applying Parseval's identity for Fourier transforms to the elements of the coefficient matrix of this system of equations, these elements can be efficiently obtained in the spectral domain in terms of the two-dimensional discrete Fourier's transforms of the Floquet-periodic basis functions $\mathbf{J}_{i}^{l}(x, y)$ ( $\left.i=1, \ldots, N_{b} ; l=1,2\right)$, and in terms of samples of the two-dimensional Fourier transform of the non periodic dyadic Green's function, $\overline{\mathbf{G}}[4]$. Once the coefficients $d_{i}^{l}$ are determined, the elements of $\overline{\mathbf{S}}_{X Y}^{\mathrm{sc}}$ can be easily obtained in terms of $d_{i}^{l}$, and in terms of the Fourier transforms of $\mathbf{J}_{i}^{l}(x, y)$ and $\overline{\mathrm{G}}$.

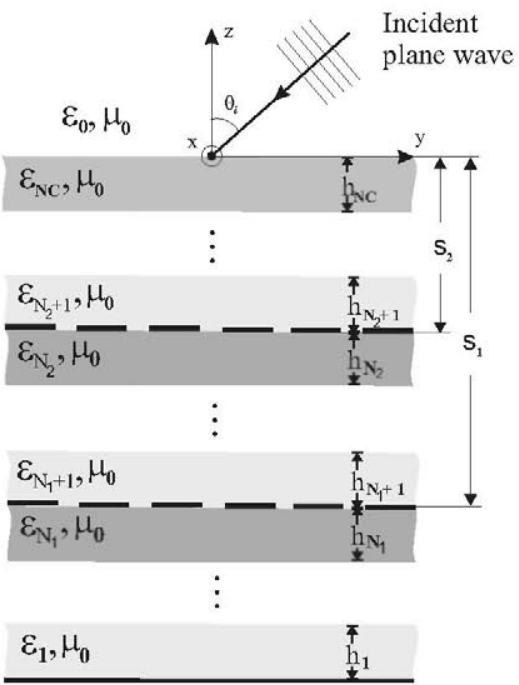

(a)

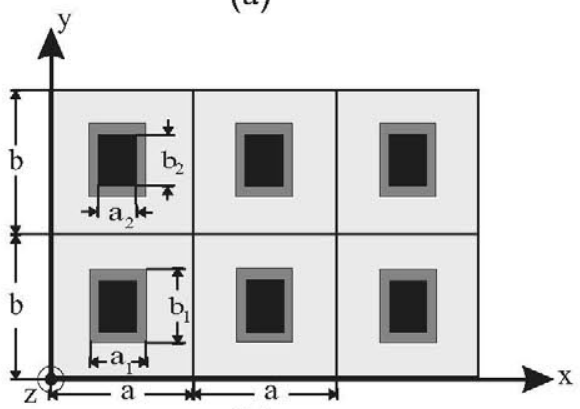

(b)

Fig. 1. Multilayered periodic structure with two stacked rectangular patches in the unit cell. The periodic structure is illuminated by a plane wave. (a) Side view. (b) Top view.

In this paper, we have used in (3) two different sets of basis functions for the approximation of the current density on the rectangular patches. One of the sets of the basis functions consists of products of sinusoidal functions as shown below [6, eqn. 26]

$$
\begin{aligned}
& \mathbf{J}_{i}^{l}(x, y)= \\
& \mathbf{J}_{r s x}^{l}=\frac{1}{b_{l}} \sin \left[\frac{\pi r}{a_{l}}\left(x-x_{0 l}\right)\right] \cos \left[\frac{\pi s}{b_{l}}\left(y-y_{0 l}\right)\right] \hat{\mathbf{x}} \\
& \quad\left(r=1, \ldots, M_{x} ; s=0, \ldots, N_{x} ; i=1, \ldots, M_{x}\left(N_{x}+1\right)\right.
\end{aligned}
$$

$\mathbf{J}_{M_{x}\left(N_{x}+1\right)+i}^{l}(x, y)=$

$\mathbf{J}_{r s y}^{l}=\frac{1}{a_{l}} \cos \left[\frac{\pi r}{a_{l}}\left(x-x_{0 l}\right)\right] \sin \left[\frac{\pi s}{b_{l}}\left(y-y_{0 l}\right)\right] \hat{\mathbf{y}}$

$$
\left(r=0, \ldots, M_{x} ; s=1, \ldots, N_{x} ; i=1, \ldots, N_{x}\left(M_{x}+1\right)\right)
$$

where $2 M_{x} N_{x}+M_{x}+N_{x}=N_{b}, x_{0 l}=\left(a-a_{l}\right) / 2$ and $y_{0 l}=\left(b-b_{l}\right) / 2(l=1,2)$. These basis function stand for the current densities of the resonant modes of rectangular 
microstrip patches in the frame of the magnetic wall cavity model. Their two-dimensional discrete Fourier's transforms can be obtained in closed form in terms of elementary functions. The other set of basis functions consists of products of Chebyshev polynomials weighted by functions that account for edge singularities as shown below [6, eqn. 25]

$$
\begin{gathered}
\mathbf{J}_{i}^{l}(x, y)=\mathbf{J}_{r s x}^{l}=\frac{1}{b_{l}} U_{r}\left[\frac{2\left(x-\frac{a}{2}\right)}{a_{l}}\right] \sqrt{1-\left[\frac{2\left(x-\frac{a}{2}\right)}{a_{l}}\right]^{2}} \\
\times \frac{T_{s}\left[\frac{2\left(y-\frac{b}{2}\right)}{b_{l}}\right]}{\sqrt{1-\left[\frac{2\left(y-\frac{b}{2}\right)}{b_{l}}\right]^{2}} \hat{\mathbf{x}}} \\
\left(r=0, \ldots, M_{x}-1 ; s=0, \ldots, N_{x} ; i=1, \ldots, M_{x}\left(N_{x}+1\right)\right) \\
\mathbf{J}_{M_{x}\left(N_{x}+1\right)+i}^{l}(x, y)=\mathbf{J}_{r s y}^{l}=\frac{1}{a_{l}} \frac{T_{r}\left[\frac{2\left(x-\frac{a}{2}\right)}{a_{l}}\right]}{\sqrt{1-\left[\frac{2\left(x-\frac{a}{2}\right)}{a_{l}}\right]^{2}}} \\
\times U_{s}\left[\frac{2\left(y-\frac{b}{2}\right)}{b_{l}}\right] \sqrt{1-\left[\frac{2\left(y-\frac{b}{2}\right)}{b_{l}}\right]^{2}} \hat{\mathbf{y}} \\
\left(r=0, \ldots, M_{x} ; s=0, \ldots, N_{x}-1 ; i=1, \ldots, N_{x}\left(M_{x}+1\right)\right)
\end{gathered}
$$

where $2 M_{x} N_{x}+M_{x}+N_{x}=N_{b}$, and $T_{i}[\cdot]$ and $U_{i}[\cdot]$ are respectively Chebyshev polynomials of first and second kind and i-th degree. The two-dimensional discrete Fourier's transforms of the basis functions in (6) and (7) can be expressed in terms of Bessel functions.

Figs. 2(a) and 2(b) show the convergence of the phase of $S_{x x}$ (which is a key parameter in the design of a reflectarray antenna) with respect to the number of basis functions $N_{b}$ of (3) for the periodic structure of Fig. 1 in the case where the stacked patches are located in a four-layered substrate. Whereas the results of Fig. 2(a) are for medium size patches ( $a_{1}=b_{1}=7.9 \mathrm{~mm}, a_{2}=b_{2}=0.7 a_{1}$ ), the results of Fig. 2(b) are for large patches with dimensions close to the dimensions of the unit cell $\left(a_{1}=b_{1}=11.5 \mathrm{~mm}, a_{2}=b_{2}=0.7 a_{1}\right)$. Note convergence within $2^{\circ}$ in the phase of $S_{x x}$ is achieved for $N_{b}=20$ in Figs. 2(a) and 2(b) when edge singularity basis functions are used. In the case of sinusoidal basis functions, convergence within $5^{\circ}$ in the phase of $S_{x x}$ is achieved when $N_{b}=40$ and $N_{b} \simeq 140$ in Figs. 2(a) and 2(b) respectively. Since the convergence values obtained with sinusoidal basis functions are still $10^{\circ}$ above the convergence values obtained with edge singularities basis functions, substantially larger values of $N_{b}$ would have been required to obtain convergence within $2^{\circ}$ in the phase of $S_{x x}$ with sinusoidal basis functions. This clearly indicates that the performance of edge singularities basis functions is much superior to that of sinusoidal basis functions concerning the convergence of the phase of $S_{x x}$ with respect to $N_{b}$. Numerical simulations have shown that whereas the convergence of $\overline{\mathbf{S}}_{X Y}$ with respect to $N_{b}$ is roughly independent of the dimensions of the patches $a_{i}$ and $b_{i}(i=1,2)$ when edge singularity basis functions are used, the value of $N_{b}$ required for convergence increases as $a_{i}$ and $b_{i}(i=1,2)$ become closer to the dimensions of the periodic cell $a$ and $b$ when sinusoidal basis functions are used. This increase in the value of $N_{b}$ required for convergence unavoidably leads to an increase in the CPU time required for the MoM-SD analysis of the periodic structure as shown in Fig. 2(c). According to this latter figure, the ratio between the CPU time required by sinusoidal basis functions (to reach convergence within $5^{\circ}$ in the phase of $S_{x x}$ ) and the CPU time required by edge singularity basis functions (to reach convergence within $2^{\circ}$ in the phase of $S_{x x}$ ) can be larger than 10 when the size of the patches is sufficiently large. The results of Fig. 2(c) indicates that important CPU time reductions may be achieved in the analysis of the periodic structure of Fig. 1 when edge singularity basis functions are used instead of sinusoidal basis functions.

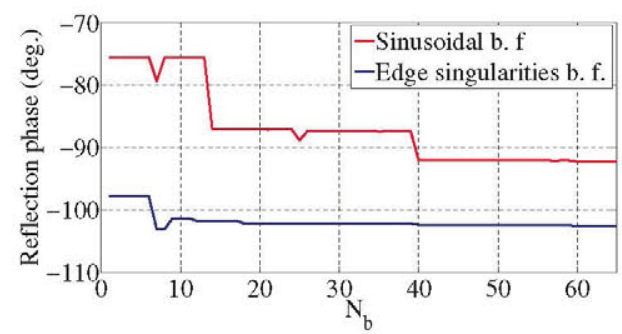

(a)

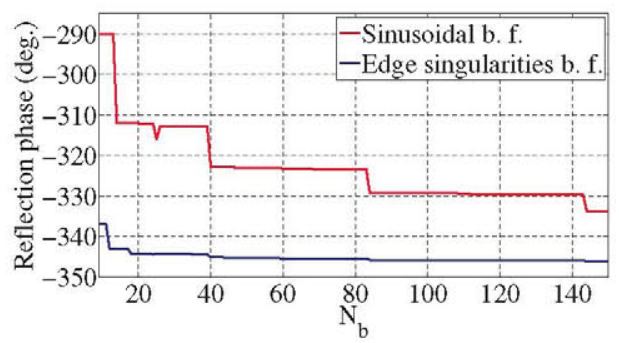

(b)

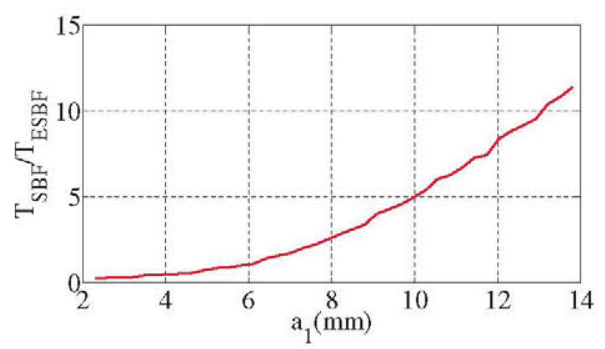

(c)

Fig. 2. (a) and (b) Convergence of the phase of $S_{x x}$ with respect to the number of basis functions $N_{b}$ for sinusoidal basis functions and edge singularity basis functions: $a_{1}=b_{1}=7.9 \mathrm{~mm}$ in (a) and $a_{1}=b_{1}=11.5 \mathrm{~mm}$ in (b). (c) Ratio between the CPU times required by sinusoidal basis functions $T_{S B F}$ and edge singularity basis functions $T_{S B F}$. Parameters: $a=b=$ $14 \mathrm{~mm} ; f=12 \mathrm{GHz} ; a_{1}=b_{1} ; a_{2}=b_{2}=0.7 a_{1} ; \theta_{i}=0^{\circ} ; N_{1}=2 ; N C=$ $N_{2}=4 ; \varepsilon_{r 1}=\varepsilon_{r 3}=1.07 ; \tan \delta_{1}=\tan \delta_{3}=0.001 ; h_{1}=h_{3}=3 \mathrm{~mm}$; $\varepsilon_{r 2}=\varepsilon_{r 4}=2.55 ; \tan \delta_{2}=\tan \delta_{4}=0.003 ; h_{2}=h_{4}=0.2 \mathrm{~mm}$.

For some cases of reflectarray design, the phase of $S_{x x}$ $\left(S_{y y}\right)$ is required in a large range of values of the geometrical dimension $a_{1}\left(b_{1}\right)$ for fixed values of the ratios $a_{1} / b_{1}, b_{2} / b_{1}$ 
and $a_{2} / a_{1}, a, b$, frequency and incidence angle [2]. Therefore, the CPU time required for the repeated analysis of the periodic structure of Fig. 1 in the design of a reflectarray can be further reduced if $S_{x x}\left(S_{y y}\right)$ is interpolated in terms of $a_{1}\left(b_{1}\right)$. In particular, in this paper we propose that $S_{x x}$ is approximately fitted as rational functions of $a_{1}$ as shown below

$$
S_{x x}=\frac{C_{0, x x}+C_{1, x x}\left(a_{1}\right)+\ldots+C_{N, x x}\left(a_{1}\right)^{N}}{1+D_{1, x x}\left(a_{1}\right)+\ldots+D_{M, x x}\left(a_{1}\right)^{M}}
$$

where the coefficients $C_{0, x x}, \ldots, C_{N, x x}, D_{1, x x}, \ldots, D_{M, x x}$ are complex numbers which are extracted from a small number of full-wave MoM-SD analysis of the periodic structure by means of a least square scheme with singular value decomposition [9]. An interpolating expression similar to (8) is proposed for $S_{y y}$ in terms of $b_{1}$.

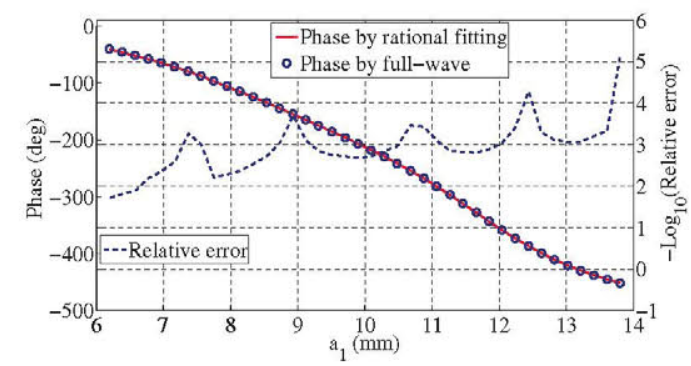

(a)

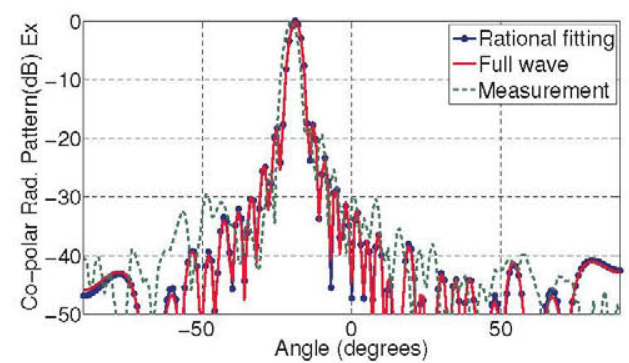

(b)

Fig. 3. (a) Phase of $S_{x x}$ obtained by means of both full-wave MoM-SD analysis and eqn. (8), and errors between the two sets of results. Parameters: $a=b=14 \mathrm{~mm} ; f=12 \mathrm{GHz} ; a_{1}=b_{1} ; a_{2}=b_{2} ; a_{2}=0.7 a_{1} ; \theta_{i}=0^{\circ}$; $N_{1}=1 ; N C=N_{2}=2 ; \varepsilon_{r 1}=\varepsilon_{r 2}=1.05 ; \tan \delta_{1}=\tan \delta_{2}=0.001$; $h_{1}=h_{2}=3 \mathrm{~mm} ; N=2 ; M=3$. (b) Comparison between numerical results (full-wave MoM-SD and eqn. (8)) and measured results for the radiation pattern of the reflectarray studied in [2] $(f=11.95 \mathrm{GHz}$.)

In Fig. 3(a) we show the results obtained for the phase of $S_{x x}$ (patches of Fig. 1 located in a two-layered substrate) with the purely numerical MoM-SD approach and with the closedform rational fitting expression of (8) in the case where $N=2$ and $M=3$ (rational function with two zeros and three poles). Note that there is a good agreement between the two sets of results, and that the relative error is on average around $0.1 \%$. In Fig.3(b) we show numerical and experimental results for the radiation pattern of a pencil beam reflectarray designed in [2]. According to Fig. 3(b), the results obtained for the radiation patterns with the full-wave MoM-SD approach and with the rational fitting approach of (8) are essentially the same. Also, good agreement is found between the numerical results and the experimental results above $-30 \mathrm{~dB}$ [2]. The ratio between the CPU times required for the total design of the reflectarray antenna is $T_{\text {full-wave }} / T_{\text {rational fitting }}=7.8$, which indicates that the rational fitting approach of (8) leads to important CPU time savings when designing a reflectarray antenna.

\section{CONCLUSIONS}

An efficient numerical tool has been implemented for the analysis of multilayered periodic structures containing embedded stacked rectangular patches in the unit cell. This numerical tool is intended to be massively used in the design of reflectarray antennas under the local periodicity assumption. The numerical tool makes use of the Method of Moments in the Spectral Domain (MoM-SD) based on multilayered Green's functions (MGF). It has been demonstrated that the use of basis functions with edge singularities in the approximation of the current density on the patches makes it possible to optimize the efficiency in the application of the MoM-SD. Also, a rational fitting technique has been introduced to obtain closed-form interpolations of the scattering parameters of the multilayered periodic structures in terms of the dimensions of the rectangular patches. It has been proved that these closedform interpolations may lead to important CPU time savings in reflectarray design.

\section{ACKNOWLEDGMENT}

This work has been supported by "Junta de Andalucía" (project TIC-4595), and by the Spanish Ministry of Science and Innovation (projects CICYT TEC2010-17567 and CONSOLIDER CSD2008-68).

\section{REFERENCES}

[1] J. Huang, J. A. Encinar, Reflectarray antennas. Piscataway, NJ/New York: IEEE Press/Wiley, 2008.

[2] J. A. Encinar, "Design of two-layer printed reflectarrays using patches of variable size," IEEE Trans. Antennas Propagat., vol. 49, pp. 1403-1410, Oct. 2001.

[3] J. A. Encinar, L. Datashvili, J. A. Zornoza, M. Arrebola, M. SierraCastañer, J. L. Besada, H. Baier, H. Legay, "Dual-polarization dualcoverage reflectarray for space spplications," IEEE Trans. on Antennas and Propag., Vol. 54, No. 10, pp. 2828-2837, Oct. 2006.

[4] R. Mittra, C. H. Chan, and T. Cwik, "Techniques for analyzing frequency selective surfaces-A review", Proc. IEEE. vol. 76, no. 12, pp. 1593-1615, Dec. 1988.

[5] F. Mesa, R. Marqués, and M. Horno, "A general algorithm for computing the bidimensional spectral Green's dyads in multilayered complex bianisotropic media: the equivalent boundary method," IEEE Trans. Microwave Theory Tech., vol. 39, pp. 1640-1649, Sept. 1991.

[6] W. C. Chew, and Q. Liu, "Resonance frequency of a rectangular microstrip patch," IEEE Trans. Antennas Propagat., vol. 36, pp. 1045-1056, Aug. 1988.

[7] M. García Vigueras, J. L. Gómez Tornero, G. Goussetis, J. S. Gómez Díaz and A. Álvarez Melcón, "A modified pole-zero technique for the synthesis of waveguide leaky-wave antennas loaded with dipole-based FSS", IEEE Trans. Antennas Propagat., vol. 58, no. 6, pp. 1971-1979, June 2010.

[8] J. L. Tsalamengas "Interaction of electromagnetic waves with general bianisotropic slabs", IEEE Trans. Microwave Theory Tech., vol. MTT40, pp. $1870-1878$, Oct. 1992.

[9] R. S. Adve, T. K. Sarkar, S. M. Rao, E. K. Miller, D. R. Pflug, “Application of the Cauchy method for extrapolating/interpolating narrowband system responses", IEEE Trans. Microwave Theory Tech., vol. 45, pp. 837-845, May 1997. 